\title{
Fibra dietética e gordura saturada: relação entre marcadores bioquímicos da síndrome metabólica
}

\author{
Dietary fiber and saturated fat: relation between biochemical markers \\ of metabolic syndrome
}

\author{
Bruna Angelo Vieira', Débora da Silva Rosenhaim², Carla Haas Piovesan' ${ }^{3}$, David Santos de Freitas ${ }^{4}$, \\ Luísa Rihl Castro ${ }^{5}$, Fabrício Edler Macagnan ${ }^{6}$, Ana Maria Pandolfo Feoli ${ }^{7}$ 西 \\ 1 Nutricionista graduada pela Pontifícia Universidade Católica do Rio Grande do Sul. Especialista em Nutrição Clínica com ênfase nas Doenças Cardiovasculares, \\ Diabetes e Obesidade pelo Instituto de Cardiologia do Rio Grande do Sul. Mestranda em Epidemiologia pela Universidade Federal do Rio Grande do Sul. \\ Nutricionista graduada pela Pontifícia Universidade Católica do Rio Grande do Sul. Especialista em Saúde da Família pelo Instituto de \\ Educação e Pesquisa - Hospital Moinhos de Vento. \\ ${ }^{3}$ Nutricionista graduada pela Pontifícia Universidade Católica do Rio Grande do Sul. Mestre em Medicina e Ciências da Saúde pela \\ Pontifícia Universidade Católica do Rio Grande do Sul. \\ ${ }^{4}$ Biólogo graduado pela Pontifícia Universidade Católica do Rio Grande do Sul. Mestre em Biociências (Zoologia) pela Pontifícia Universidade Católica \\ do Rio Grande do Sul. Doutorando em Biologia pela Universidade do Vale do Rio dos Sinos. \\ ${ }_{5}^{5}$ Nutricionista graduada pela Universidade do Vale do Rio dos Sinos. Mestre em Ciências Médicas pela Universidade Federal do Rio Grande do Sul. \\ ${ }^{6}$ Fisioterapeuta graduado pela Universidade de Cruz Alta. Especialista em Fisioterapia Músculo-Esquelética pela Universidade de Cruz Alta. \\ Mestre em Ciências Biológicas (Fisiologia) pela Universidade Federal do Rio Grande do Sul. Doutor em Clínica Médica e Ciências da Saúde pela \\ Pontifícia Universidade Católica do Rio Grande do Sul. \\ Nutricionista graduada pelo Instituto Metodista de Educação e Cultura. Mestre em Ciências Biológicas (Bioquímica) pela Universidade Federal \\ do Rio Grande do Sul. Doutora em Ciências Biológicas (Bioquímica) pela Universidade Federal do Rio Grande do Sul.
}

Este estudo foi conduzido com recursos da Fundação de Amparo à Pesquisa do Estado do Rio Grande do Sul (FAPERGS) e do Conselho Nacional de Desenvolvimento Científico e Tecnológico (CNPq).

\section{RESUMO}

Objetivos: Relacionar o consumo de fibra alimentar e de gordura saturada com marcadores bioquímicos em indivíduos portadores de síndrome metabólica.

Métodos: A base de dados deste estudo transversal foi formada por adultos de ambos os sexos com diagnóstico de síndrome metabólica conforme o National Cholesterol Education Program 's Adult Treatment Panel III, participantes de um estudo realizado na Pontifícia Universidade Católica do Rio Grande do Sul. Foram utilizados os dados de todos os indivíduos cadastrados na linha de base que possuíam informações completas sobre o consumo alimentar. O consumo de fibra alimentar e de gordura saturada foi apurado a partir das informações obtidas de um recordatório alimentar 24 horas e de um registro alimentar de dois dias. Foram avaliados os sujeitos de forma geral e estratificados por sexo. A análise de dados foi realizada por estatística descritiva e teste $t$ de Student. A correlação entre o consumo de fibras e de gordura saturada com marcadores bioquímicos foi obtida através do coeficiente de correlação de Pearson. Foi utilizado nível de significância de 5\%.

Resultados: Foram encontrados 79 indivíduos cujos registros continham as informações completas, sendo incluídos no estudo. Observou-se correlação positiva significativa do consumo de gordura saturada com níveis séricos de triglicerídeos $(r=0,30 ; p=0,008)$ e com insulina ( $r=0,26$; $\mathrm{p}=0,021)$. Além disso, observou-se correlação inversa significativa entre consumo de fibras e níveis séricos de HDL-colesterol $(\mathrm{r}=-0,28$; $\mathrm{p}=0,011$ ). Quando estratificados os indivíduos por sexo, observou-se que a correlação positiva entre consumo de gordura saturada e nível de triglicerídeos manteve-se significativa apenas entre os homens $(\mathrm{r}=0,44 ; \mathrm{p}=0,034)$.

Conclusões: Nessa população com diagnóstico de síndrome metabólica, foram encontrados níveis mais baixos de HDL-colesterol nos indivíduos que referiam maior consumo de fibra alimentar, enquanto os indivíduos que relataram elevado consumo de gordura saturada tiveram níveis mais altos de insulina e de triglicerídeos. Na análise estratificada por sexo, correlação positiva foi encontrada apenas entre consumo de gordura saturada e níveis séricos de triglicerídeos em homens.

DESCRITORES: síndrome X metabólica; fibras alimentares; ácidos graxos; marcadores bioquímicos; HDL-colesterol.

\section{ABSTRACT}

Aims: To relate the consumption of dietary fiber and saturated fat to biochemical markers in individuals with metabolic syndrome.

Methods: The database of this cross-sectional study consisted of both male and female adults diagnosed with metabolic syndrome, according to the National Cholesterol Education Program's Adult Treatment Panel III, who participated in a study at the Pontifical Catholic University of Rio Grande do Sul. Data on all individuals registered at baseline who had complete information about their food intake were used. Consumption of dietary fiber and saturated fat was calculated based on the information provided by a 24-hour recall survey and a two-day food record. We evaluated the subjects in general and stratified by gender. The data were analyzed using descriptive statistics and the Student's $t$ test. The correlation between fiber and saturated fat intake and biochemical markers was measured by Pearson's correlation coefficient. The significance level was set at $5 \%$. 
Results: There were 79 individuals whose records contained complete information and who were eventually included in the study. A significant positive correlation was observed between saturated fat intake and serum triglyceride levels $(\mathrm{r}=0.30 ; \mathrm{p}=0.008)$ and between saturated fat intake and insulin $(\mathrm{r}=0.26 ; \mathrm{p}=0.021)$. Also, a significant inverse correlation was observed between fiber intake and serum levels of HDL cholesterol $(\mathrm{r}=-0.28 ; \mathrm{p}=0.011)$. When stratified by gender, the positive correlation between consumption of saturated fat and triglycerides remained significant only among men $(\mathrm{r}=0.44 ; \mathrm{p}=0.034)$.

Conclusions: In this population diagnosed with metabolic syndrome, lower levels of HDL cholesterol were found in individuals who referred higher intake of dietary fiber, while individuals who reported elevated consumption of saturated fat had higher levels of insulin and triglycerides. In the gender-stratified analysis, positive correlation was found only between saturated fat consumption and serum triglyceride levels in men.

KEY WORDS: metabolic syndrome X; dietary fiber; fatty acids; biochemical markers; HDL-cholesterol.

\section{INTRODUÇÃO}

A síndrome metabólica (SM) é um complexo conjunto de distúrbios associados aos fatores de risco de doença cardiovascular, principalmente resistência à insulina e obesidade abdominal [1]. Segundo estudos, a prevalência da SM nos Estados Unidos está estimada entre $22,9 \%$ e $26,7 \%$ [2,3], enquanto na AméricaLatina a prevalência oscila entre $25 \%$ e $45 \%$ [4]. No Brasil, um estudo realizado em Porto Alegre revelou alta prevalência $(61,5 \%)$ de SM [5], conforme os critérios National Cholesterol Education Program's Adult Treatment Panel III (NCEP-ATP III) [6]. Esses mesmos critérios foram utilizados em outra pesquisa desenvolvida no Rio de Janeiro, na qual a prevalência foi de $55,6 \%$ [7].

Diversos fatores podem contribuir para o aparecimento da SM. Dentre eles estão aumento de peso, tabagismo e sedentarismo, além de fatores dietéticos $[8,9]$. O plano alimentar indicado na SM recomenda a ingestão de alimentos integrais com baixo índice glicêmico e adequação no consumo de fibra alimentar solúvel $[1,6]$. De acordo com Marlett et al., fibras solúveis são eficazes na regulação dos níveis plasmáticos de glicose, colesterol e triglicerídeos, constituindo fator preventivo de doença aterosclerótica e diabetes mellitus [10].

Porém, são controversas as relações existentes entre consumo de fibra alimentar e SM. Há evidências de que dietas ricas em grãos integrais, frutas e verduras estão associadas a uma prevalência mais baixa de SM, enquanto que dietas à base de alimentos refinados, frituras e refrigerantes indicam um aumento na ocorrência da mesma [11,12]. Entretanto, estudos demonstram não haver associação entre o consumo de fibra alimentar e alguns componentes da SM, tais como pressão arterial, colesterol ligado a lipoproteínas de alta densidade (HDL-c), glicemia de jejum e triglicerídeos [13,14].
A terapia nutricional preconizada pelo NCEPATP III consiste na redução do consumo de gorduras saturadas [6], uma vez que essas gorduras constituem o principal fator determinante do aumento das concentrações plasmáticas de colesterol ligado a lipoproteínas de baixa densidade (LDL-c) e estão relacionadas à alteração na ação da insulina [15]. Além disso, a sensibilidade insulínica está inversamente associada à ingestão de gordura pela qualidade dos ácidos graxos e pela relação positiva com o peso corporal [1]. O efeito dos ácidos graxos é variável, diminuindo a secreção de insulina de acordo com o grau de insaturação e elevando com o aumento da cadeia de carbonos. Assim, os ácidos graxos de cadeia longa aumentam a secreção insulínica, como resposta aos níveis basais de glicose [15].

Sendo assim, o objetivo do presente estudo foi relacionar o consumo de fibra alimentar e de gordura saturada com marcadores bioquímicos (glicemia de jejum, insulina e perfil lipídico) de indivíduos portadores de SM.

\section{MÉTODOS}

Trata-se de um estudo transversal descritivo [16], realizado em base de dados de um estudo principal, "Efeito da modificação do estilo de vida sobre os fatores de risco cardiovascular que compõem os critérios de diagnóstico da síndrome metabólica, marcadores inflamatórios e balanço autonômico: um estudo randomizado", realizado na Pontifícia Universidade Católica do Rio Grande do Sul (PUCRS).

Foram selecionados todos os indivíduos cadastrados na linha de base que possuíam suas informações completas sobre o consumo alimentar e exames bioquímicos. A base de dados é formada por voluntários, com idade entre 30 e 60 anos, de ambos os sexos, com diagnóstico de SM, conforme os critérios 
diagnósticos adotados pelo NCEP-ATP III, e que aceitaram participar da pesquisa perante a assinatura do Termo de Consentimento Livre e Esclarecido. Os critérios do NCEP-ATP III para diagnóstico da SM incluem a associação de três ou mais dos componentes a seguir: circunferência abdominal para homens $>102 \mathrm{~cm}$ e mulheres $>88 \mathrm{~cm}$; triglicerídeos $\geq 150 \mathrm{mg} / \mathrm{dl}$; HDL-c para homens $<40 \mathrm{mg} / \mathrm{dl}$ e mulheres $<50 \mathrm{mg} / \mathrm{dl}$; pressão arterial sistólica $\geq 130 \mathrm{mmHg}$ ou diastólica $\geq 85 \mathrm{mmHg}$; e glicemia em jejum $\geq 110 \mathrm{mg} / \mathrm{dl}$ [6].

Foi respeitada a confidencialidade e o sigilo das informações coletadas no estudo, segundo a Resolução 196/96 do Conselho Nacional de Saúde-Ministério da Saúde que estabelece as Diretrizes e Normas Regulamentadoras Sobre Pesquisas Envolvendo Seres Humanos [17]. O estudo foi aprovado pelo Comitê de Ética e Pesquisa da PUCRS, sob o processo $\mathrm{n}^{\mathrm{o}}$ 06/03024. Todos os voluntários receberam, no momento da adesão ao estudo primário e de forma individual, informações referentes aos procedimentos aos quais seriam submetidos, através do Termo de Consentimento Livre e Esclarecido, que foi assinado pelos indivíduos que aceitaram participar, autorizando a disponibilização e utilização de seus dados para a pesquisa.

Os voluntários passaram por avaliações antropométricas, físicas e nutricionais realizadas no Centro de Reabilitação da PUCRS. Os exames bioquímicos foram realizados no Laboratório de Análises Clínicas do Hospital São Lucas da PUCRS. As análises bioquímicas foram realizadas através de amostras de sangue coletadas por punção venosa após 12 horas de jejum. Glicose plasmática, colesterol total, triglicerídeos séricos e concentrações séricas de HDL-c foram medidos por métodos enzimáticos que utilizam reagentes no analisador totalmente automatizado Vitros 950 sistema de química seca (Johnson \& Johnson, Rochester, NY). O LDL-c foi estimado usando a equação de Friedewald [18]. Os valores de insulina foram obtidos através de um sistema automatizado, usando a técnica de quimiluminescência ADVIA (Centaur Immunoassay System, Siemens).

Foram utilizados os dados de idade, peso e altura dos participantes, para posterior cálculo do índice de massa corporal (IMC); e valores de circunferência abdominal, triglicerídeos, HDL-c, LDL-c, colesterol total, pressão arterial, glicemia em jejum e insulina. O consumo de fibra alimentar e de gordura saturada foi apurado a partir das informações obtidas de um recordatório alimentar 24 horas e de um registro alimentar de dois dias. O recordatório alimentar 24 horas foi utilizado para obter informações sobre a ingestão alimentar das últimas 24 horas antes das consultas. Durante a primeira ida dos sujeitos ao centro de pesquisa, o recordatório alimentar 24 horas foi aplicado por equipe previamente treinada e certificada. Após a realização desse recordatório, os participantes foram instruídos a como proceder para realização do registro alimentar pelo método prospectivo de avaliação do consumo alimentar, onde os alimentos ingeridos são mensurados e descritos (com maior detalhamento de ingredientes e métodos de preparo). A mensuração foi realizada por porções estimadas e medidas caseiras padronizadas. Por se tratar de um registro alimentar de dois dias, os indivíduos deveriam preencher os mesmos durante um dia de final de semana e outro dia a livre escolha (de segunda a sexta-feira). A ingestão dos itens alimentares de interesse foi mensurada através do Programa de Apoio à Nutrição - Calculadora Nutricional ${ }^{\mathbb{R}}$ (Nutwin), 2002 (versão 1.5.2.8). Foi utilizada como referência, em relação à ingestão de fibra alimentar, a I Diretriz Brasileira de Diagnóstico e Tratamento da Síndrome Metabólica, que recomenda consumo de 20 a $30 \mathrm{~g} /$ dia [1].

A análise dos dados foi realizada através de estatística descritiva (média e desvio padrão) e teste t de Student. A normalidade dos dados foi avaliada através do teste de Kolmogorov-Smirnov. A correlação entre o consumo de fibra alimentar e de gordura saturada com os valores dos marcadores bioquímicos (triglicerídeos, colesterol total, HDL-c, LDL-c, glicemia em jejum e insulina) foi obtida através do coeficiente de correlação de Pearson, onde foi considerado $\mathrm{r}=0,10$ até 0,30 (correlação fraca); $r=0,40$ até 0,60 (correlação moderada); $r=0,70$ até 1 (correlação forte) [19]. Foi utilizado nível de significância de $5 \%$.

\section{RESULTADOS}

Foram identificados 79 indivíduos cujos registros possuíam todas as informações necessárias para este estudo, sendo 23 do sexo masculino e 56 do sexo feminino. Os dados clínico-laboratoriais apresentaramse de forma homogênea para todas as variáveis consideradas, com exceção do peso $(\mathrm{p}=0,002)$, da altura $(p<0,001)$ e da circunferência abdominal $(p=0,034)$, que se mostraram superiores nos homens, o que é esperado na comparação entre os sexos, e dos níveis de HDL-c $(p<0,001)$, os quais indicaram diferença significativa, estando mais baixos em homens do que em mulheres. Não houve diferença de consumo de gordura saturada e fibra alimentar entre os sexos (Tabela 1). 
Tabela 1. Caracterização da amostra, composta por indivíduos com diagnóstico de síndrome metabólica.

\begin{tabular}{|c|c|c|c|c|}
\hline \multirow[b]{2}{*}{ Características } & \multicolumn{3}{|c|}{ Média \pm Desvio Padrão } & \multirow[b]{2}{*}{$\mathbf{P}^{*}$} \\
\hline & $\begin{array}{c}\text { Total } \\
n=79\end{array}$ & $\begin{array}{c}\text { Homens } \\
n=23\end{array}$ & $\begin{array}{c}\text { Mulheres } \\
\mathrm{n}=56\end{array}$ & \\
\hline Idade (anos) & $50,7 \pm 6,6$ & $49,6 \pm 8,2$ & $51,1 \pm 5,9$ & 0,367 \\
\hline Peso (kg) & $87,2 \pm 12,8$ & $93,9 \pm 11,6$ & $84,4 \pm 12,3$ & $0,002^{+}$ \\
\hline Altura (m) & $1,61 \pm 0,08$ & $1,69 \pm 0,07$ & $1,58 \pm 0,06$ & $<0,001^{+}$ \\
\hline Índice de Massa Corporal (kg/m²) & $33,4 \pm 4,0$ & $32,5 \pm 3,6$ & $33,7 \pm 4,2$ & 0,232 \\
\hline Circunferência Abdominal (cm) & $106 \pm 9$ & $109 \pm 7$ & $105 \pm 9$ & $0,034^{+}$ \\
\hline Triglicerídeos (mg/dL) & $206 \pm 108$ & $242 \pm 100$ & $191 \pm 109$ & 0,059 \\
\hline HDL-colesterol (mg/dL) & $44 \pm 10$ & $38 \pm 8$ & $46 \pm 9$ & $<0,001^{+}$ \\
\hline Glicemia de Jejum (mg/dL) & $108 \pm 37$ & $107 \pm 31$ & $108 \pm 40$ & 0,948 \\
\hline Pressão Arterial Sistólica (mmHg) & $131 \pm 15$ & $134 \pm 13$ & $130 \pm 16$ & 0,387 \\
\hline Pressão Arterial Diastólica (mmHg) & $83 \pm 11$ & $84 \pm 10$ & $82 \pm 11$ & 0,525 \\
\hline Consumo de Fibra Alimentar (g) & $16,5 \pm 6,0$ & $18,5 \pm 5,5$ & $15,7 \pm 6,0$ & 0,057 \\
\hline Consumo de Gordura Saturada (g) & $22,0 \pm 10,1$ & $23,9 \pm 10,9$ & $21,1 \pm 9,8$ & 0,276 \\
\hline
\end{tabular}

* Teste t de Student para comparação por sexo.

${ }^{+}$Diferença significativa entre os sexos.
Observou-se correlação positiva significativa entre elevado consumo de gordura saturada e altos níveis séricos de triglicerídeos $(\mathrm{r}=0,30 ; \mathrm{p}=0,008)$ e de insulina $(\mathrm{r}=0,26 ; \mathrm{p}=0,021)$. Além disso, observou-se correlação inversa significativa entre consumo de fibras e níveis séricos de HDL-c ( $\mathrm{r}=-0,28 ; \mathrm{p}=0,011)$, ou seja, quanto maior o consumo de fibra alimentar menores os níveis de HDL-c (Tabela 2). No entanto, quando feita a estratificação por sexo, manteve-se significativa apenas a correlação entre consumo de gordura saturada e triglicerídeos em homens $(\mathrm{r}=0,44 ; \mathrm{p}=0,034)$ (Tabela 3). Não houve correlação significativa entre as demais variáveis pesquisadas.

Tabela 2. Correlação entre marcadores bioquímicos, gordura saturada e fibras em 79 indivíduos com diagnóstico de síndrome metabólica.

\begin{tabular}{lccc}
\multicolumn{1}{c}{ Marcadores bioquímicos } & Gordura Saturada & Fibras \\
Colesterol Total & r & 0,04 & $-0,12$ \\
Triglicerídeos & $\mathrm{P}$ & 0,758 & 0,300 \\
& $\mathrm{r}$ & $0,30^{*}$ & 0,05 \\
HDL-colesterol & $\mathrm{P}$ & 0,008 & 0,666 \\
\multirow{2}{*}{ LDL-colesterol } & $\mathrm{r}$ & $-0,17$ & $-0,28^{*}$ \\
& $\mathrm{P}$ & 0,146 & 0,011 \\
Glicemia de Jejum & $\mathrm{r}$ & $-0,07$ & $-0,10$ \\
& $\mathrm{P}$ & 0,532 & 0,363 \\
Insulina & $\mathrm{r}$ & $-0,07$ & $-0,10$ \\
& $\mathrm{P}$ & 0,566 & 0,369 \\
\hline
\end{tabular}

r: Coeficiente de correlação de Pearson.

* Correlação significativa.
Tabela 3. Correlação entre marcadores bioquímicos, gordura saturada e fibras em 79 indivíduos com diagnóstico de síndrome metabólica, estratificados por sexo.

\begin{tabular}{|c|c|c|c|}
\hline \multicolumn{2}{|c|}{ Marcadores bioquímicos } & Gordura Saturada & Fibras \\
\hline \multicolumn{4}{|l|}{ HOMENS $(n=23)$} \\
\hline \multirow[t]{2}{*}{ Colesterol Total } & $\mathrm{r}$ & 0,12 & 0,03 \\
\hline & $\mathrm{p}$ & 0,586 & 0,909 \\
\hline \multirow[t]{2}{*}{ Triglicerídeos } & $r$ & $0,44^{*}$ & 0,00 \\
\hline & $\mathrm{p}$ & $0,034^{*}$ & 0,987 \\
\hline \multirow[t]{2}{*}{ HDL-colesterol } & $r$ & 0,09 & $-0,14$ \\
\hline & $\mathrm{p}$ & 0,672 & 0,517 \\
\hline \multirow[t]{2}{*}{ LDL-colesterol } & r & $-0,26$ & 0,08 \\
\hline & $\mathrm{p}$ & 0,236 & 0,733 \\
\hline \multirow[t]{2}{*}{ Glicemia de Jejum } & $\mathrm{r}$ & 0,18 & $-0,20$ \\
\hline & $\mathrm{p}$ & 0,401 & 0,367 \\
\hline \multirow[t]{2}{*}{ Insulina } & $r$ & 0,38 & 0,44 \\
\hline & $\mathrm{p}$ & 0,076 & 0,633 \\
\hline \multicolumn{4}{|l|}{ MULHERES $(n=56)$} \\
\hline \multirow[t]{2}{*}{ Colesterol Total } & $r$ & 0,03 & $-0,13$ \\
\hline & $\mathrm{p}$ & 0,819 & 0,325 \\
\hline \multirow[t]{2}{*}{ Triglicerídeos } & r & 0,21 & 0,01 \\
\hline & $\mathrm{p}$ & 0,113 & 0,964 \\
\hline \multirow[t]{2}{*}{ HDL-colesterol } & $r$ & $-0,22$ & $-0,25$ \\
\hline & $\mathrm{p}$ & 0,109 & 0,064 \\
\hline \multirow[t]{2}{*}{ LDL-colesterol } & $r$ & $-0,02$ & $-0,02$ \\
\hline & $\mathrm{p}$ & 0,907 & 0,907 \\
\hline \multirow[t]{2}{*}{ Glicemia de Jejum } & $r$ & $-0,15$ & $-0,15$ \\
\hline & $\mathrm{p}$ & 0,262 & 0,262 \\
\hline \multirow[t]{2}{*}{ Insulina } & $\mathrm{r}$ & 0,17 & 0,17 \\
\hline & $\mathrm{p}$ & 0,221 & 0,221 \\
\hline
\end{tabular}

r: Coeficiente de correlação de Pearson.

* Correlação significativa. 


\section{DISCUSSÃO}

O colesterol sérico pode ser reduzido através da diminuição da absorção de colesterol pelo intestino, em função da viscosidade da fibra solúvel, e pela capacidade que esse tipo de fibra tem de aumentar a eliminação de sais biliares nas fezes, pois, como mecanismo de compensação, o fígado produz ácidos biliares a partir da degradação de colesterol. As fibras solúveis são muito eficazes para controle/regulação de níveis de triglicerídeos, glicose e colesterol [20]. Entretanto, resultados semelhantes não foram observados no presente estudo, tendo em vista que as correlações encontradas não foram estatisticamente significativas, mesmo após estratificação por sexo.

A ação hipolipemiante das fibras solúveis tem sido relatada em múltiplos estudos. Uma metanálise agrupou 12 estudos os quais comparavam indivíduos que consumiam altos teores de fibra alimentar com um grupo controle com ingestão sem suplementação de fibras. Aqueles que tinham um maior consumo de fibra dietética obtiveram taxas significativamente menores de LDL-c e triglicerídeos em comparação com o grupo controle. Em relação ao HDL-c, não foi observada diferença entre grupos. Contudo, Glore et al., citados nessa metanálise, relatam que em dietas com elevada ingestão de fibras solúveis as concentrações de HDL-c podem diminuir entre $3 \%$ e $20 \%$ [21]. O mesmo pode ser constatado nos resultados deste estudo, visto que os níveis plasmáticos de HDL-c dos participantes também se mostraram diminuídos naqueles que tiveram um maior teor de fibra na sua dieta. É importante salientar que esta redução não é favorável para o indivíduo, uma vez que níveis baixos de HDL-c são forte e independentemente associados com maior risco de desenvolvimento de doença cardíaca [22,23]. Porém, o consumo de fibras não deve ser desencorajado, tendo em vista os benefícios relacionados ao seu consumo, tais como promoção de perda de peso e menor resistência insulínica [24,25], que são alguns dos principais fatores ligados ao desenvolvimento de SM [1].

A correlação entre consumo de gordura saturada e níveis séricos de insulina pode estar relacionada à resistência insulínica, resultando em hiperinsulinemia. As alterações na resistência à insulina não dependem exclusivamente da adiposidade, mas também estão intimamente ligadas à ingestão de gordura, não apenas pela quantidade, mas pelo tipo de ácido graxo consumido, principalmente os saturados. Uma das explicações é que a redução na ação da insulina seria causada pela modificação no perfil lipídico da membrana celular. Esta alteração ocorre devido ao elevado consumo de gordura saturada, levando à diminuição na atividade de enzimas (dessaturases) que fazem parte da biossíntese de ácidos graxos. Esse processo causa uma maior disponibilidade desse tipo de gordura, o que altera a composição das membranas celulares, deixando-as com maior grau de saturação, acarretando também prejuízo na transmissão do sinal insulínico que ativa a captação da glicose. Assim sendo, quanto mais saturada é a membrana fosfolipídica, maior é a resistência à insulina presente no tecido envolvido [26,27].

Fatores exógenos, como dietas ricas em gorduras saturadas, podem elevar a produção de quilomicron e colesterol ligado a lipoproteínas de muito baixa densidade (VLDL-c), ocasionando hipertrigliceridemia [28]. Ácidos graxos saturados são apontados como importantes fatores de origem alimentar na elevação dos níveis séricos de colesterol e triglicerídeos [29]. Uma dieta com grande quantidade de gordura saturada, quando comparada à dieta com alto teor de ácidos graxo monossaturados, tende a aumentar níveis de triglicerídeos, principalmente no período pósprandial [30]. O fato da gordura saturada influenciar de forma positiva nas concentrações plasmáticas dos triglicerídeos corrobora a relação notada neste estudo, onde quanto maior o consumo de gordura saturada maior o nível de triglicerídeos quando avaliada a população em geral e, quando estratificado, apenas nos homens.

As limitações deste estudo se referem à análise de inquéritos dietéticos e tamanho amostral. Apesar de ser um instrumento muito utilizado em estudos epidemiológicos, o recordatório alimentar 24 horas tem limitações, pois depende da memória do entrevistado (na análise dos dados há dificuldades inerentes à identificação correta dos alimentos, bem como à quantificação das receitas e preparações). Além disso, há limitações nos programas para a análise química dos alimentos da dieta, baseados em fontes disponíveis, mas, por vezes, pouco confiáveis ou incompletas em termos de nutrientes, sobretudo os micronutrientes [31]. Para minimizar erros, os pesquisadores verificaram o correto preenchimento dos registros e utilizaram dois recordatórios (um de dia de semana e outro de final de semana). Em relação ao delineamento, os dados foram obtidos com um desenho transversal, que não permite realizar uma inferência sobre causalidade e o tamanho da amostra pode explicar a falta de associações significativas.

Os achados do presente estudo corroboram dados encontrados na literatura, contribuem para o 
esclarecimento do assunto e reforçam a necessidade de maiores investigações quanto ao consumo de fibras e gorduras saturadas em indivíduos com diagnóstico de SM. Neste estudo, esse consumo relacionou-se de forma significativa com os marcadores bioquímicos triglicerídeos, insulina e HDL-c. Foram encontrados níveis mais baixos de HDL-c nos indivíduos que referiam maior consumo de fibra alimentar, enquanto os indivíduos que relataram elevado consumo de gordura saturada tiveram níveis mais altos de insulina e de triglicerídeos. Na análise estratificada por sexo, uma correlação positiva foi encontrada apenas entre consumo de gordura saturada e níveis séricos de triglicerídeos em homens.

\section{REFERÊNCIAS}

1. Sociedade Brasileira de Cardiologia. I Diretriz Brasileira de Diagnóstico e Tratamento da Síndrome Metabólica. Arq Bras Cardiol. 2005;84(I). http://dx.doi.org/10.1590/S0066-782X2005000700001

2. Beltrán-Sánchez H, Harhay MO, Harhay MM, McElligott S. Prevalence and trend of Metabolic Syndrome in the adult US population, 1999-2010. J Am Coll Cardiol. 2013; 62(8): 697-703. http://dx.doi.org/10.1016/j.jacc.2013.05.064

3. Ford ES, Giles WH, Mokdad AH. Increasing prevalence of the metabolic syndrome among U.S. adults. Diabetes Care. 2004;27(10): 2444-449. http://dx.doi.org/10.2337/diacare.27.10.2444

4. López-Jaramillo P, Sánchez RA, Diaz M, Cobos L, Bryce A, Parra-Carrillo JZ, Lizcano F, Lanas F, Sinay I, Sierra ID, Peñaherrera E, Bendersky M, Schimid H, Botero R, Urina M, Lara J, Foss MC, Márquez G, Harrap S, Ramírez AJ, Zanchetti A, em nome do Grupo de Especialistas da América Latina. Consenso Latino-Americano de Hipertensão em Pacientes com Diabetes Tipo 2 e Síndrome Metabólica. Arq Bras Endocrinol Metab. 2014;58(3):205-25. http://dx.doi.org/10.1590/0004-2730000003019

5. Bopp M, Barbiero S. Prevalence of Metabolic Syndrome in Outpatients of the Institute of Cardiology of Rio Grande do Sul. Arq Bras Cardiol. 2009;93(5):439-42. http://dx.doi.org/10.1590/S0066-782X2009001100006

6. Third Report of the National Cholesterol Education Program (NCEP) Expert Panel on Detection, Evaluation, and Treatment of High Blood Cholesterol in Adults (Adult Treatment Panel III) Final Report. Circulation. 2002;106(25):3143-421.

7. Leão LSCS, Barros EG, Koifman RJ. Prevalência de Síndrome Metabólica em Adultos Referenciados para Ambulatório de Nutrição no Rio de Janeiro, Brasil. Rev Bras Cardiol. 2010;23(2):93-100.

8. Dima-Cozma C, Gavrilută C, Mitrea G, Cojocaru DC. The importance of healthy lifestyle in modern society: a medical, social and spiritual perspective. Eur J Sci Theol. 2014;10(3):111-20.

9. Perk J, Backer GD, Gohlke H, Graham I, Reiner Z, Verschuren WMM, et al. European Guidelines on cardiovascular disease prevention in clinical practice (version 2012). The Fifth Joint Task Force of the European Society of Cardiology and Other Societies on Cardiovascular Disease Prevention in Clinical Practice (constituted by representatives of nine societies and by invited experts). Eur Heart J. 2012; 33: 1635-1701. http://dx.doi.org/10.1093/eurheartj/ehs092

10. Marlett JA, McBurney MI, Slavin JL. Position of the American Dietetic Association: Health Implications of Dietary Fiber. J Am Diet Assoc. 2002;102(7):993-1000. http://dx.doi.org/10.1016/S0002-8223(02)90228-2

11. Lutsey PL, Steffen LM, Stevens J. Dietary Intake and the Development of the Metabolic Syndrome: the Atherosclerosis Risk in Communities Study. Circulation. 2008;117(6):754-61. http://dx.doi.org/10.1161/CIRCULATIONAHA.107.716159

12. McKeown NM, Meigs JB, Liu S, Saltzman E, Wilson PW, Jacques PF. Carbohydrate Nutrition, Insulin Resistance, and the Prevalence of the Metabolic Syndrome in the Framingham Offspring Cohort. Diabetes Care. 2004;27(2):538-46. http://dx.doi.org/10.2337/ diacare.27.2.538

13. Veldhuis L, Koppes LL, Driessen MT, Samoocha D, Twisk JW. Effects of Dietary Fibre Intake during Adolescence on the Componentes of the Metabolic Syndrome at the age of 36 years: the Amsterdam Growth and Health Longitudinal Study. J Hum Nutr Diet. 2010;23(6): 601-8. http://dx.doi.org/10.1111/j.1365-277X.2010.01089

14. Dall'Alba V, Silva FM, Antonio JP, Steemburgo T, Royer CP, Almeida JC, Gross JL, Azevedo MJ. Improvement of the Metabolic Syndrome Profile by Soluble Fibre - Guar Gum - in Patients with Type 2 Diabetes: a Randomised Clinical Trial. Br J Nutr. 2013;110(9):1601-10. http://dx.doi.org/10.1017/S0007114513001025

15. Santos CRB, Portella ES, Ávila SS, Soares EA. Fatores Dietéticos na Prevenção e Tratamento de Comorbidades Associadas à Síndrome Metabólica. Rev Nutr. 2006;19(3):389-401. http://dx.doi.org/10.1590/S1415-52732006000300010

16. Goldim JR. Manual de Iniciação à Pesquisa em Saúde. 2ª ed. Porto Alegre: Dacasa Editora; 2000.

17. Brasil. Ministério da Saúde. Conselho Nacional de Saúde. Resolução no 196/96 sobre Pesquisa Envolvendo Seres Humanos. Bioética. Brasília: Ministério da Saúde; 1996.

18. Friedewald WT, Levy RI, Fredrickson DS. Estimation of the Concentration of Low-Density Lipoprotein Cholesterol in Plasma, without Use of the Preparative Ultracentrifuge. Clin Chem. 1972;18(6):499-502.

19. Dancey C, Reidy J. Estatística sem Matemática para Psicologia: Usando Spss para Windows. Porto Alegre: Artmed; 2006.

20. Gonçalves MCR, Costa MJC, Asciutti LSR, Diniz MFFM. Fibras Dietéticas Solúveis e suas Funções nas Dislipidemias. Rev Bras Nutr Clin. 2007;22(2):167-73. 
21. Olson BH, Anderson SM, Becker MP, Anderson JW, Hunninghake DB, Jenkins DJA, LaRosa JC, Rippe JM, Roberts DCK, Stoy DB, Summerbell CD, Truswell AS, Wolever TMS, Morris DH, Fulgoni III VL. Psyllium-Enriched Cereals Lower Blood Total Cholesterol and LDL Cholesterol, but not HDL Cholesterol, in Hypercholesterolemic Adults: Results of a Meta-Analysis. J Nutr. 1997;127(10):1973-80.

22. Rubins HB, Robins SJ, Collins D, Fye CL, Anderson JW, Elam MB, Faas FH, Linares E, Schaefer EJ, Schectman G, Wilt TJ, Wittes J. Gemfibrozil for the Secondary Prevention of Coronary Heart Disease in Men with Low Levels of High-Density Lipoprotein Cholesterol. N Engl J Med. 1999;341(6):410-8. http://dx.doi.org/10.1056/NEJM199908053410604

23. Birjmohun RS, Hutten BA, Kastelein JJP, Stroes ESG. Efficacy and Safety of High-Density Lipoprotein Cholesterol-Increasing Compounds: A Meta-Analysis of Randomized Controlled Trials. J Am Coll Cardiol. 2005;45(2):185-97. http://dx.doi.org/10.1016/j.jacc.2004.10.031

24. Mello VD, Laaksonen DE. Fibras na Dieta: Tendências Atuais e Benefícios à Saúde na Síndrome Metabólica e no Diabetes Melito Tipo 2. Arq Bras Endocrinol Metab. 2009;53(5):509-18. http://dx.doi.org/10.1590/S0004-27302009000500004

25. Liu S, Willett WC, Manson JE, Hu FB, Rosner B, Colditz G. Relation Between Changes in Intakes of Dietary Fiber and Grain Products and Changes in Weight and Development of Obesity Among Middle-Aged Women. Am J Clin Nutr. 2003;78(5):920-7.

26. Pereira LO, Francischi RP, Júnior AHL. Obesidade: Hábitos Nutricionais, Sedentarismo e Resistência à Insulina. Arq Bras Endocrinol Metab. 2003;47(2):111-27. http://dx.doi.org/10.1590/S0004-27302003000200003

27. Wajchenberg BL, Santomauro ATMG, Nery M, Santos RF, Silva MELR, Ursich MJM, Rocha DM. Resistência à Insulina: Métodos Diagnósticos e Fatores que Influenciam a Ação da Insulina. Arq Bras Endocrinol Metab. 1999;43(2):76-85. http://dx.doi.org/10.1590/ S0004-27301999000200003

28. Xavier HT, Izar MC, Faria Neto JR, Assad M H, Rocha VZ, Sposito AC, Fonseca FA, dos Santos JE, Santos RD, Bertolami MC, Faludi AA, Martinez TLR, Diament J, Guimarães A, Forti NA, Moriguchi E, Chagas ACP, Coelho OR, Ramires JAF. V Diretriz Brasileira de Dislipidemias e Prevenção da Aterosclerose. Arq Bras Cardiol. 2013; 101(4Supl.1): 1-22.

29. Leaf DA. Hypertriglyceridemia: A Guide to Assessment and Treatment. Hospital Physycian. 2008; 17-23,32.

30. Reiner Z, Catapano AL, De Backer G, Graham I, Taskinen MR, Wiklund O, Agewall S, Alegría E, Chapman MJ, Durrington P, Erdine S, Halcox J, Hobbs RH, Kjekshus JK, Perrone Filardi P, Riccardi G, Storey RF, David W; Clinical Practice Guidelines Committee of the Spanish Society of Cardiology. ESC/EAS Guidelines for the management of dyslipidaemias. Eur Heart J. 2011; 32:1769-1818. http://dx.doi.org/10.1093/eurheartj/ehr158

31. Cuppari L, Anção MS. Uso de programas computadorizados na avaliação dietética. In: Fisberg RM, Slater B, Marchioni DML, Martini LA. Inquéritos alimentares: métodos e bases científicos. São Paulo: Manole; 2005. p. 71-82. 